\title{
Absent Pulmonary Valve Syndrome - Prenatal Diagnosis and Postnatal Correlation with a Review of Literature
}

\author{
Brinda Sabu, Tazeen Khan, Vidyalekshmy Ranganayaki \\ Department of High risk Pregnancy and Perinatology, KIMS Health, Trivandrum, India \\ Email address: \\ brinda_sabu@yahoo.co.in (B. Sabu)
}

\section{To cite this article:}

Brinda Sabu, Tazeen Khan, Vidyalekshmy Ranganayaki. Absent Pulmonary Valve Syndrome - Prenatal Diagnosis and Postnatal Correlation with a Review of Literature. International Journal of Clinical and Experimental Medical Sciences. Vol. 7, No. 3, 2021, pp. 70-73.

doi: $10.11648 / j$.jijcems.20210703.13

Received: April 25, 2021; Accepted: May 10, 2021; Published: June 25, 2021

\begin{abstract}
Absent Pulmonary valve syndrome (APVS) or congenital absence of pulmonary valve is a rare conotruncal anomaly in which the pulmonary valve is absent, dysplastic or rudimentary. It can be isolated or associated with tetralogy of Fallot and has a favourable to guarded postnatal outcome according to the associations. In this case report, we discuss an interesting case of APVS with postnatal correlation. Methods: Detailed USG including fetal echocardiography was performed on an ultrasound machine using appropriate presets. The abdominal and cardiac situs were normal. Segmental evaluation of the fetal heart along with color and spectral doppler was performed. Results: The fetus had the typical features of APVS, with a dilated pulmonary artery and branches, a subaortic ventricular septal defect, overriding of the aorta, and agenesis of ductus arteriosus. There was typical to and fro flow noted on color and spectral Doppler imaging representing forward stenotic and retrograde regurgitant flow. Postnatal Echocardiogram confirmed our antenatal findings. Though the baby underwent surgical correction, he succumbed due to the effects of multiorgan dysfunction and the underlying chromosomal defect. Conclusions: A dedicated fetal echocardiography around 22 weeks can accurately diagnose the condition due to its typical features of dilated main and branched pulmonary arteries, absent /dysplastic pulmonary valves with color and spectral doppler revealing features of stenosis and insufficiency. However the outcome of fetuses in APVS with additional anomalies is guarded due to the heart defect itself, respiratory complications and associated chromosomal abnormalities.
\end{abstract}

Keywords: Absent Pulmonary Valve Syndrome, Ductal Agenesis, Patent Ductus Arteriosus, Tetralogy of Fallot, Tricuspid Atresia, Ventricular Septal Defect

\section{Introduction}

APVS is a rare conotruncal anomaly in which the pulmonary valve is absent, dysplastic or rudimentary $[1,2]$. In $30 \%$ of cases, the ductus arteriosus (DA) is absent, the pulmonary annulus is stenotic allowing to and fro blood flow across the dysplastic pulmonary valve, leading to severe dilatation of the pulmonary trunk and its branches. The dilated main pulmonary artery and the branches gives rise to the Bow tie sign. [3] Association with 22q11 microdeletion in APVS is also noted. [4] The presence of DA causes severe pulmonary regurgitation leading to congestive heart failure and fetal loss. [5] Severely dilated pulmonary arteries and associated cardiomegaly cause bronchial compression resulting in bronchomalacia. This leads to respiratory compromise at birth and chronic obstructive lung disease later in life. Thus APVS is associated with significant perinatal morbidity and mortality. [6]

APVS is classified in two categories: (a) Absent pulmonary valve with VSD also known as Fallot type (b) Absent pulmonary valve with intact ventricular septum also known as Non Fallot type. Widely accepted theory for Fallot type APVS is that agenesis of DA contribute to the aneurysmal dilatation of the main and the branched pulmonary arteries. [7]. In non-Fallot type APVS, since the DA is present, aorta and main pulmonary artery accommodate bulk of increased flow, thus sparing the branched pulmonary arteries.

Prenatal APVS accounts for $15-20 \%$ of all fetuses with TOF and $0.2-0.4 \%$ of all congenital cardiac defects [8]. Due to the rarity of APVS, information on the clinical presentation and outcome of prenatally diagnosed cases is 
scarce. Large fetal case series report fetal and postnatal survival rates of $<20 \%$. The high loss rate was related to pregnancy termination, fetal heart failure, respiratory disease and chromosomal abnormalities $[9,10]$.

\section{Case Report}

33 years old $\mathrm{G}_{3} \mathrm{P}_{1} \mathrm{~L}_{0} \mathrm{~A}_{1}$ in a nonconsanguineous marriage was referred to us in view of abnormal cardiac findings by ultrasound at 36 weeks of GA. Her previous baby had $\mathrm{TOF} /$ pulmonary atresia and succumbed three weeks after birth. No genetic evaluation of the baby or the parents was done.

A detailed USG along with fetal echocardiogram was done. Cardiac and abdominal situs were normal. Systemic and pulmonary venous drainage appeared normal. Cardiomegaly was noted. Atrioventricular and ventriculoarterial concordance was noted. Chamber asymmetry (Right $>$ Left) with subaortic type VSD was noted. [Figure 1] DA was absent. Grossly dilated main pulmonary artery (PA) and branched pulmonary arteries [Figure 2] with dysplastic pulmonary valve [Figure 3] giving rise to the typical 'bow tie' appearance was noted. Doppler interrogation across pulmonary valve showed severe stenosis [Figure 4] with PSV of $250 \mathrm{~cm} / \mathrm{sec}$. To and fro flow across the pulmonary valves indicating pulmonary valve stenosis with regurgitation was also noted. A diagnosis of TOF/ APVS /Absent DA was made.

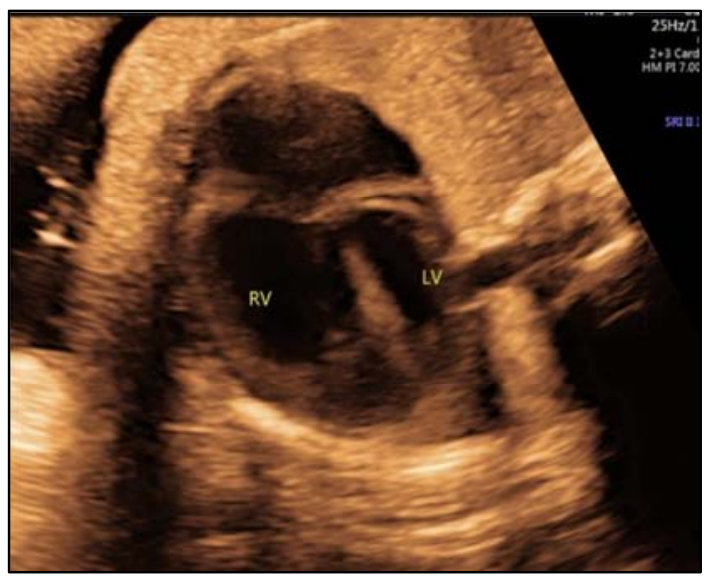

Figure 1. Basal 4 chamber view. Cardiomegaly with Chamber asymmetry (Rt $>$ Lt) Note the VSD.

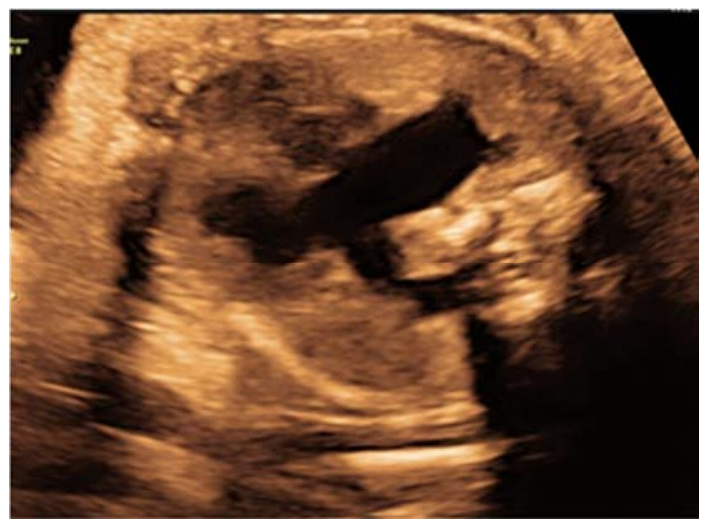

Figure 2. Dilated Main, right and left pulmonary arteries, "Bow-Tie sign”.

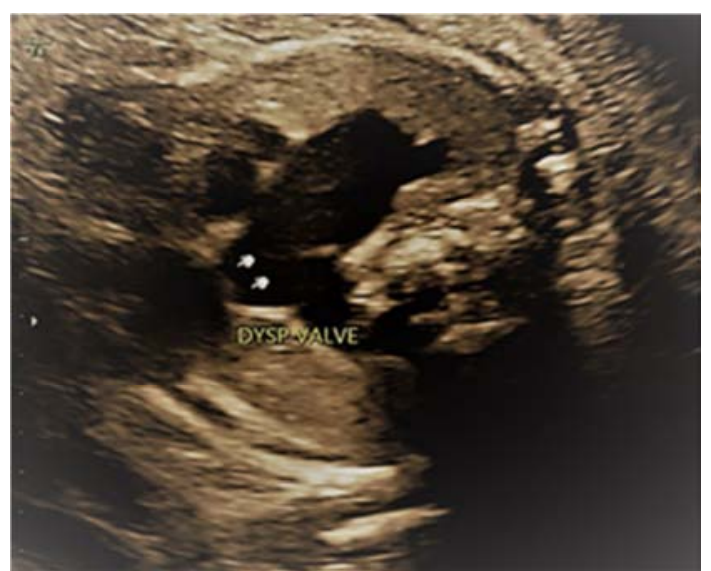

Figure 3. Dilated PAs with dysplastic pulmonary valve.

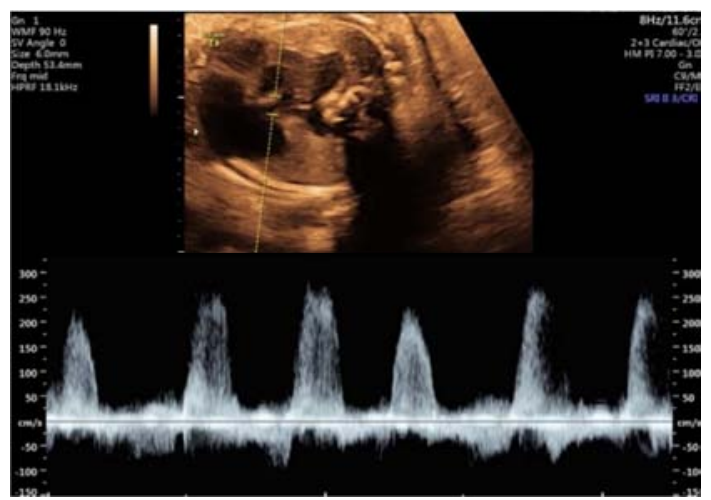

Figure 4. High pressure gradient of $250 \mathrm{~cm} / \mathrm{sec}$ across pulmonary valve.

In view of cardiac anomaly in the previous pregnancy and the recurrence of same in the current pregnancy, the association of chromosomal/genetic syndromes were explained to the couple. Possible intrauterine complications such as congestive heart failure and fetal hydrops and the importance of detailed postnatal genetic evaluation of the baby was also stressed upon. Pediatric cardiology and neonatology consults were sought.

A male baby of $2.25 \mathrm{~kg}$ was delivered by term LSCS, who cried soon after birth with APGAR scores of $7 \& 8$ at $1 \& 5$ minutes respectively. The heart rate was $60 \mathrm{bpm}$, had good perfusion and was maintaining saturation of $70-80 \%$ off oxygen. Postnatal ECHO findings were: Right ventricular hypertrophy and dilatation, perimembranous VSD with 75\% aortic override, dysplastic pulmonary valve with pressure gradient of $55 \mathrm{mmHg}$ across the pulmonary valve and pulmonary regurgitation. Main pulmonary artery, left and right pulmonary arteries were dilated (RPA $>$ LPA). Features were suggestive of TOF with absent pulmonary valve syndrome, thus confirming the antenatal diagnosis.

Postnatally though the baby did not require acute care, subsequently he developed arrhythmias along with signs of hypoparathyroidism \& hypocalcemia and was suspected to have Velocardiofacial syndrome. Microarray analysis confirmed microdeletion of chromosome 22q11. 2. (Velocardiofacial syndrome).

The baby was readmitted on the $23^{\text {rd }}$ day with respiratory failure, was intubated and put on mechanical ventilation. X- 
ray evaluation showed asymmetric lungs with mechanical obstruction of left bronchus and compensatory hyper inflation of left lung. He underwent Pulmonary arterioplasty the next day, but sadly succumbed 2 weeks postsurgery due to low cardiac output and multiorgan dysfunction.

\section{Discussion}

APVS can be suspected when we image absent/dysplastic pulmonary valves with malaligned VSD and associated echocardiographic findings like aneurysmal dilatation of pulmonary arteries and the branches appearing as 'bow tie' or 'balloon' like hypoechoic shadow.[3] The presence of both stenotic and regurgitant Doppler gradient at the pulmonary annulus differentiates APVS from uncomplicated TOF.

According to current consensus, there are three variants of APVS: the predominant type of APVS with TOF and agenesis of the DA; and two rare non TOF variants including APVS with an intact ventricular septum and a patent DA, and APVS with an intact ventricular septum and membranous tricuspid atresia. [11]

Pulmonary complications are more commonly seen in Fallot type of APVS due to the large aneurysmal pulmonary arteries causing bronchomalacia and if untreated leads to death due to intractable right heart failure. In the non Fallot type, intrauterine hydrops and fetal death occurs in $7-15 \%$ during the second half of gestation. If they survive delivery, early cardiorespiratory failure can occur if not surgically intervened. Surgical treatment includes closure of VSD and pulmonary arterioplasty done in early infancy.

An interstitial deletion on chromosome 22 and associated CATCH syndrome (cardiac anomalies, abnormal facies, thymic hypoplasia, cleft palate, and hypocalcemia) have been reported in fetuses with Fallot type APVS. [7]

According to the study by Razavi et al [6] in 2003, among 20 cases of APVS, 6 neonates died even after surgery, and only 3 survived more than 1 year suggesting poor surgical results for APVS than comparable variants with tetralogy of Fallot. [12] Furthermore, the prognosis was not significantly affected by whether the disease is diagnosed prenatally or postnatally and none of the echocardiographic variables, including pulmonary valve annulus, right pulmonary artery diameter and cardiomegaly, was a significant predictor of neonatal respiratory distress and adverse outcome. [13]

Later in 2013, a single centre study by Wertaschnigg et al reported that prenatally detected isolated APVS with absent DA had improved survival rates of $>80 \%$ in liveborn infants with active postnatal care. (10) as compared to; their counterparts with additional anomalies. However our baby inspite of having a successful surgery succumbed and this explains the bad outcome of APVS with additional anomalies.

A systematic review of 36 articles done by Abuhamad et al in 2015 compared the postnatal outcomes, genetic testing results, and sonographic findings in 3 subtypes of tetralogy of Fallot and inferred that the survival rates in TOF with APVS is significantly less than TOF with pulmonary stenosis.[14] In Gottschalk's study (2017) where a retrospective analysis of 40 fetuses over a period of 13 years was done, there were only $27.5 \%$ survivors. However they noted that APVS with isolated TOF and agenesis of the DA has a better outcome of $>80 \%$ than those with additional anomalies which was comparable to the study by Wertaschnigg et al. Patency of the DA, reversed flow in ductus venosus, umbilical artery or middle cerebral artery, and hydrops/increased nuchal translucency thickness were significantly associated with non-survival. [11]

\section{Conclusion}

There is updated evidence that agenesis of DA is essential for the survival in fetuses with TOF and APVS. With a patent DA, the increased volume load precludes the survival of fetus with APVS beyond the early second trimester. Mid trimester scan has a low detection rate in diagnosing APVS, probably owing to the incomplete expression of the full disease spectrum. But a dedicated fetal echocardiography around 22 weeks can accurately diagnose the condition. Though APVS with isolated TOF and agenesis of DA has a better prognosis with low morbidity with adequate postnatal care, the outcome of the fetuses with APVS with additional anomalies is poor because of respiratory distress, the heart defect itself and associated chromosomal abnormalities.

\section{Conflicts of Interest}

The authors declare that they have no competing interests.

\section{References}

[1] Lev M, Eckner FA. The pathologic anatomy of tetralogy of Fallot and its variations. Dis Chest 1964; 45: 251-261.

[2] Emmanouilides GC, Thanopoulos B, Siassi B, Fishbein M. "Agenesis" of ductus arteriosus associated with the syndrome of tetralogy of fallot and absent pulmonary valve. Am J Cardiol 1976; 37: 403-409.

[3] S. Philip, M. Varghese, K. Manohar and K. M. Cherian, Absent pulmonary valve syndrome: prenatal cardiac ultrasound diagnosis with autopsy correlation European Journal of Echocardiography (2011) 12, E44 doi: 10.1093/ejechocard/jer155.

[4] Sleurs E, De Catte L, Benatar A. Prenatal diagnosis of absent pulmonary valve syndrome in association with 22q11 deletion. J Ultrasound Med 2004; 23: 417-422.

[5] Yeager SB, Van Der Velde ME, Waters BL, Sanders SP. Prenatal role of the ductus arteriosus in absent pulmonary valve syndrome. Echocardiography 2002; 19: 489-493.

[6] Razavi RS, Sharland GK, Simpson JM. Prenatal diagnosis by echocardiogram and outcome of absent pulmonary valve syndrome. Am J Cardiol 2003; 91: 429-432.

[7] Joshi Alpana N, Rane Hema S, Kamble Rajesh, Mestry Pravin J, Hemal Maniar, Shah Yatin. Prenatal diagnosis of absent pulmonary valve syndrome. J Ultrsound Med. 2010; 29: 823829. 
[8] Allan LD, Sharland GK, Milburn A, Lockhart SM, Groves AM, Anderson RH, Cook AC, Fagg NL. Prospective diagnosis of 1,006 consecutive cases of congenital heart disease in the fetus. J Am Coll Cardiol 1994; 23: 1452-1458.

[9] Volpe P, Paladini D, Marasini M, Buonadonna AL, Russo MG, Caruso G, Marzullo A, Arciprete P, Martinelli P, Gentile M. Characteristics, associations and outcome of absent pulmonary valve syndrome in the fetus. Ultrasound Obstet Gynecol 2004; 24: 623-628.

[10] D. Wertaschnigg, M. Jaeggi, D. Chitayat, P. Shannon, G. Ryan, M. Thompson, S. J. Yoo And E. Jaeggi, Prenatal diagnosis and outcome of absent pulmonary valve syndrome: contemporary single-center experience and review of the literature Ultrasound Obstet Gynecol 2013; 41: 162-167.

[11] I. Gottschalk C. Jehle, U. Herberg J. Breuer, K. Brockmeier, G. Bennink, A. Hellmund, B. Strizek, U. Gembruch, A. Geipel, C. Berg, Prenatal diagnosis of absent pulmonary valve syndrome from first trimester onwards: novel insights into pathophysiology, associated conditions and outcome Ultrasound Obstet Gynecol 2017; 49: 637-642.
[12] Chowdhury UK, Airan B, Kumar AS, Sharma R, Bhan A, Kothari SS, Saxena A, Juneja R, Venugopal P. Management of tetralogy of Fallot with absent pulmonary valve: early and mid-term results of a uniform approach. Ind Heart J 2000; 52: $54-59$.

[13] Moon-Grady AJ, Tacy TA, Brook MM, Hanley FL, Silverman $\mathrm{NH}$. Value of clinical and echocardiographic features in predicting outcome in the fetus, infant, and child with tetralogy of Fallot with absent pulmonary valve complex. Am J Cardiol 2002; 89: 1280-1285.

[14] Yili Zhao, MD, Alfred Abuhamad, MD, Jonathan Fleenor, MD, et al, Prenatal and Postnatal Survival of Fetal Tetralogy of Fallot A Meta-analysis of Perinatal Outcomes and Associated Genetic Disorders American Institute of Ultrasound in Medicine, J Ultrasound Med 2016; 35: 905-915, 0278-4297. 